\title{
La mobilisation d'une perspective interactionniste pour éclairer la négociation du territoire de pratique des coachs en gestion
}

\author{
Sylvie Guignon, Ph.D. ${ }^{1}$
}

\author{
Université Laval
}

Joëlle Morrissette, Ph.D.

Université de Montréal

\begin{abstract}
Résumé
Le point de vue adopté pour étudier le coaching en gestion est généralement prescriptif vis-à-vis de la pratique, posant des modèles théoriques comme référents, privilégiant une approche déductive et des méthodologies quantitatives pour mesurer ses impacts en termes de retour sur investissement, de niveau de satisfaction client. Sans nier l'importance de s'interroger sur le service rendu, il peut être réducteur d'envisager la pratique à travers des indicateurs de performance décontextualisés. Y a-t-il un intérêt à renouveler le regard posé sur l'objet pour une autre compréhension de la pratique et de ses enjeux? Cette contribution s'appuie sur une recherche doctorale (2012) qui interroge le développement du coaching dans la mosaïque des professions d'accompagnement au développement professionnel de gestionnaires. Menée selon une perspective interactionniste, elle s'est attachée à recontextualiser l'émergence de cette pratique, mettant au cœur de l'interprétation les interactions qui la coconstruisent.

Mots clés

PERSPECTIVE INTERACTIONNISTE, COACHING EN GESTION, CONCEPTS SENSIBILISATEURS, NÉGOCIATION DE POINTS DE VUE, PROCESSUS D’ÉMERGENCE
\end{abstract}

\section{Introduction}

Lorsque l'on part à la découverte d'un lieu, à l'occasion d'une randonnée en montagne par exemple, on emprunte un chemin qui nous fait entrevoir petit à petit tout un paysage, complexe et changeant à chaque détour de la piste choisie. Bien souvent, il existe plusieurs sentiers que l'on peut emprunter, chacun identifié par rapport à sa spécificité, et susceptibles de conduire à des observations différentes; les points de vue choisis laissent voir des facettes

RECHERCHES QUALITATIVES - Vol. 32(2), pp. 78-102.

LA RECHERCHE QUALITATIVE DANS LES SCIENCES DE LA GESTION. DE LA TRADITION À L'ORIGINALITÉ

ISSN 1715-8702 - http://www.recherche-qualitative.qc.ca/Revue.html

(C) 2013 Association pour la recherche qualitative 
singulières du lieu en question. Certains chemins sont parcourus de nombreuses fois et deviennent des voies balisées, familières pour arpenter le lieu; mais il est toujours possible de trouver d'autres moyens de l'explorer, renouvelant l'expérience, les perspectives, la vision que l'on s'était faite de l'endroit.

Il en est de même des recherches qui sont menées au sein des disciplines. Les chercheurs qui œuvrent dans un domaine ont éprouvé des approches au fil du temps, privilégiant des méthodologies qui adressent des problématiques particulières et qui dressent un certain portrait des objets d'étude. Pour autant, il est toujours possible de renouveler le regard posé sur un sujet en sortant des sentiers battus et en proposant une approche moins familière dans le cadre d'une discipline, possiblement « classique » dans une autre.

Comme le propose l'introduction de ce numéro spécial qui invite à interroger la place croissante des approches qualitatives en sciences de la gestion, les activités professionnelles qui prennent place dans les organisations sont plus particulièrement étudiées à l'aune d'approches méthodologiques quantitatives. Cette situation peut notamment s'expliquer par la tradition de la discipline, mais aussi par la légitimation accrue du chiffre, portée notamment par le contexte de la nouvelle gestion publique (NGP) qui s'impose actuellement au Québec comme dans d'autres pays occidentaux et qui privilégie un usage instrumental de données chiffrées pour gouverner (Desrosières, 2008; Morrissette \& Legendre, sous presse). Pour autant, les approches qualitatives portent en elles le potentiel de renouveler le regard posé sur un objet qui se dérobe si l'on s'en tient aux questionnements classiquement admis dans le domaine (Parissier \& Audet, 2013).

C'est dans cette optique que cette contribution vise à présenter une recherche qualitative réalisée sur le coaching en gestion, soit une pratique visant l'accompagnement au développement professionnel de gestionnaires en entreprise, en empruntant à une perspective interactionniste comme envisagée dans la tradition de Chicago (Chapoulie, 2001). Il sera donc question, à travers le processus normalisé d'une recherche, de présenter en quoi opter pour une perspective interactionniste influence chaque étape de l'étude menée, amenant un regard compréhensif sur la pratique plutôt que prescriptif.

Une telle approche demande de poser une prémisse : adopter une perspective interactionniste comme posture générale de recherche conduit à inscrire l'objet dans le social, à le (re)contextualiser plutôt qu'à l'étudier de façon "aseptisée ", comme on le ferait dans le cadre d'une pratique de laboratoire où on a pour habitude d'isoler ce qu'on étudie (Morrissette, 2010). L'accent est alors mis sur les interactions qui lient les acteurs à travers leurs pratiques (interactionnisme), ainsi que sur les univers de signification auxquels 
ils se réfèrent (symbolique), ces significations étant elles-mêmes soumises à un processus continu d'interprétation (des définitions de la situation enracinées les unes dans les autres) (Morrissette, Guignon, \& Demazière, 2011). Il s'agit donc d'adopter un point de vue qui ne considère plus seulement l'activité des coachs en ce qu'elle est et doit être rentable, mais bien comme un ensemble de conduites sociales, d'interactions qui façonnent la pratique de ces acteurs.

Ainsi, une première partie de cette contribution fait état du contexte de l'étude en se centrant sur la variété d'acteurs qui participent du monde social dans lequel s'insère le coaching de gestion. Une deuxième partie présente le cadre théorique qui guide une lecture possible de la situation et des interactions qui façonnent le coaching, avant de considérer la question qui a orienté la recherche. Une troisième partie expose le cadre méthodologique mis en œuvre, en matière d'investigation de terrain et d'approche analytique, qui a privilégié le sens que les coachs accordent à leurs activités. Une quatrième partie propose des résultats de la recherche qui illustre le rôle des interactions dans la coconstruction de la pratique du coaching.

\section{Le cadre contextuel : une (re)contextualisation du coaching en gestion}

Pour construire un objet de recherche selon une perspective interactionniste, il convient de poursuivre une démarche de (re)contextualisation (Abbott, 1999). Pour ce faire, on s'intéresse à réaliser un schéma des liens qui peuvent exister autour de l'objet d'étude pour l'appréhender dans toute sa richesse et sa complexité. À cette fin, dans ses «ficelles du métier» de chercheur, Becker (2002) propose de considérer tous les acteurs qui peuvent être impliqués, de près ou de loin, afin de donner une vie au sujet, c'est-à-dire de le réinsérer dans le réseau social qui le construit. C'est de cette manière que le coaching en gestion a été abordé dans le cadre de la recherche (Guignon, 2012), soit à partir de différents angles d'interaction, afin de rendre compte des sources d'influence qui composent le contexte social qui sert actuellement de terreau à l'émergence de cette pratique d'accompagnement au développement professionnel. Comme on le verra, c'est dans cette perspective qu'ont été examinés plusieurs points évoqués dans la suite : le contexte socio-économique qui semble avoir favorisé l'émergence de cette pratique; les particularités qui ont conduit à l'essor d'une pluralité d'offres d'intervention; la mosaïque des pratiques qui prennent en charge le développement professionnel de gestionnaires; des manières de faire qui sont privilégiées par les praticienscoachs; l'intérêt que certains acteurs, appartenant à la communauté scientifique ou professionnelle, affichent vis-à-vis de la pratique. 


\section{Le contexte socio-économique}

Accorder une importance au contexte socio-économique des années 1980-1990 qui ont vu les prémices de la pratique, c'est mettre en relief les conditions de l'émergence de cette nouvelle offre d'accompagnement au développement professionnel. À cette époque, la prépondérance de la compétition internationale, la poursuite de la performance et de la rentabilisation des ressources (y compris humaines), et la recherche d'une différenciation locale dans un contexte de mondialisation ont caractérisé le monde des entreprises (Boltanski \& Chiapello, 1999; Crozier, 1994). Confrontées à une obligation de résultat de plus en plus pressante, ces dernières cherchent à développer le potentiel professionnel de leurs employés «clés » en misant bien sûr sur leur compétence et leur savoir-faire, mais plus encore sur leur adaptabilité à un environnement en perpétuel mouvement, leur capacité à renouveler des modes de gestion et à faire face à des défis insoupçonnés (Brunel, 2004). C'est donc dans ce contexte que les entreprises se tournent vers des interventions de coaching, y cherchant de nouveaux modèles de soutien à la performance.

\section{L'évolution de la pratique}

Faire le point sur l'évolution du coaching en gestion ouvre à la richesse du modèle d'affaires dans lequel se déploie la pratique, relevant la pluralité des offres qui s'est développée au fil du temps. Si les premières interventions de coaching se déroulaient dans le cadre d'une relation avec un coach externe et avaient pour cible des dirigeants d'entreprise (Hall, Otazo, \& Hollenbeck, 1999), la pratique se décline aujourd'hui sous de nombreuses formes et atteint de multiples clients. On parle maintenant de coaching réalisé en externe ou en interne, d'interventions individuelles ou collectives, d'activités professionnelles ou d'outils pour gestionnaires (Frisch, 2005; Malarewicz, 2002, 2003; Sperry, 2008). De la même manière, la diversité des clients qui font appel à ces praticiens s'est accrue, par exemple des gestionnaires de tous types d'entreprises ou de services publics, de commissions et d'établissements scolaires, mais aussi des particuliers (Feldman \& Lankau, 2005; Joo, 2005). Cette diversification croissante joue un rôle dans les ajustements de pratique qui ont encore lieu aujourd'hui.

\section{La mosaïque des autres pratiques}

Évoquer l'existence d'autres pratiques de soutien au développement professionnel dévoile la mosaïque de pratiques orientées vers le développement professionnel dans laquelle s'insère le coaching, amenant à considérer une combinaison de genres (Abbott, Stening, Atkins, \& Grant, 2006; Paul, 2004). Il est ainsi question de mentorat, de tutorat, de conseil, de formation, par exemple, et la concomitance de pratiques souvent partiellement définies elles- 
mêmes, conçues tantôt de façon distincte, tantôt de façon analogue, en opposition ou en complément, aboutit à dresser un portrait flou et contrasté d'un domaine en mutation, tel que le relèvent Audet et Couteret (2005) ou encore Feldman et Lankau (2005). Ces auteurs considèrent alors que cette indétermination des frontières entretiendrait une certaine confusion entre les pratiques et demande des négociations pour identifier les visées de chacune.

\section{Les manières de faire}

Cerner les multiples manières de faire du coaching instruit sur les différentes sources auxquelles puise la pratique qui parait dès lors éclectique. Les coachs réfèrent à la fois à la pédagogie, à la psychologie, au management, à leurs expériences professionnelles préalablement acquises (et pas seulement comme gestionnaire en entreprise, mais aussi comme formateur ou coach sportif par exemple), etc. (Bloch, 1996; Eaton \& Brown, 2002; Guignon, 2006). Interroger les manières de faire conduit aussi à mettre en lumière les tensions qui tissent la mise en œuvre de la pratique. Celle-ci est décrite tour à tour comme visant le développement de conduites dites performantes ou l'acquisition d'une réflexivité transposable dans l'action, comme évoluant entre intervention directive ou d'accompagnement, comme étant tantôt un outil de contrôle social, tantôt un moyen de socialisation (Guignon, 2006; Rappin, 2006; Vernazobres, 2006). Ce kaléidoscope d'images qui se superposent et qui renvoient à des représentations parfois très contrastées du coaching renseigne sur la complexité qui préside encore à la mise en œuvre d'une pratique qui n'est pas encore stabilisée et prévisible.

\section{Les intérêts concurrents}

Interroger ce qui se dit et s'écrit à propos du coaching permet de rendre compte de l'intérêt concurrent des chercheurs de deux disciplines, celles des sciences de la psychologie (Berglas, 2002; Kilburg, 2000) et de la gestion (Garman, Whiston, \& Zlatopec, 2000; Gray, 2006), qui ont des visées différentes pour une pratique considérée par les premières dans une perspective de remédiation et par les secondes dans une perspective développementale. Ces deux disciplines sont engagées dans une lutte territoriale, dirions-nous, cherchant à encadrer les pratiques de coaching et à en contrôler l'efficacité selon des modèles qui leur sont propres (Feldman \& Lankau, 2005; Guignon, 2012; Joo, 2005). À côté de cet intérêt de la communauté scientifique pour le coaching, il est aussi possible de relever la vitalité de l'offre professionnelle qui attire nombre de praticiens tels des consultants, des formateurs ou encore des thérapeutes qui tendent à s'approprier le coaching et démarchent des clients en avançant l'intérêt scientifique comme formule de vente pour leurs interventions. Pour autant, la position des coachs eux-mêmes est claire : ils se 
considèrent comme des praticiens « à part entière », ayant des visées et une pratique distincte de celle de consultants ou de thérapeutes (Arfel, 2002). Enfin, ce tour d'horizon permet d'appréhender l'intérêt des clients qui semblent considérer de plus en plus le coaching comme un moyen efficient de développement professionnel; ceux-ci deviennent alors des partenaires actifs dans la régulation de l'offre de coaching, en contribuant à en définir les modes d'action, les limites, arbitrant entre les praticiens qu'ils reconnaissent ou pas comme coachs (par exemple, Hall et al., 1999; Wasylyshyn, 2003).

Le cadre contextuel, en n'ayant pas adopté les « traditionnelles lunettes » qui prescrivent des modèles éprouvés dans d'autres situations, a délaissé la question de l'efficacité de la pratique qui attire nécessairement l'attention sur des visées de productivité et conduit à vouloir rationaliser la pratique. La lecture ici proposée, faisant le panorama de l'inscription sociale de l'objet, ramène au premier plan d'un cadre contextuel les enjeux que tissent les interactions des différents acteurs (coachs et autres praticiens, clients et prescripteurs, chercheurs) qui ont un intérêt pour le coaching. En allant au-delà de ce que peuvent proposer les recherches menées qui mettent de l'avant les canons disciplinaires, et en explorant le contexte dans lequel se déploie le coaching, l'objet se construit en intégrant différentes facettes, et sa définition s'enrichit au fur et à mesure de l'avancement de l'exploration du contexte social dans lequel il s'insère. En d'autres mots, en situant l'objet d'étude dans l'espace et le temps, en recherchant la variété des points de vue qui s'expriment, en considérant le monde social et les interactions qui lui donnent une forme, une perspective interactionniste va au-delà d'une vision lisse et décontextualisée pour appréhender un «objet échevelé», c'est-à-dire aux contours brouillés, pourvus de longues connexions, et dont les « utilisateurs » doivent être vus comme ancrés dans des contextes particuliers, engagés (Latour, 1999).

Le cadre contextuel de la recherche conduit à définir l'objet comme une profession en émergence, dont le devenir est encore incertain, une profession qui est à se construire dans et par les interactions entre membres et avec les autres groupes auxquels ils sont liés. Dans cette optique, des éclairages théoriques ont été cherchés pour rendre compte de manière dynamique des deux mouvements à l'œuvre dans ce contexte, mouvements qui sous-tendent la constitution d'un groupe professionnel et la définition de sa pratique : un mouvement d'expansion à l'externe, d'une part, et un mouvement d'organisation à l'interne, d'autre part. 


\section{Le cadre théorique : le coaching sous l'angle des interactions inter et intra groupe}

Le contexte social et scientifique évoqué précédemment suggère un monde en mouvement, dans lequel s'opère actuellement une « redistribution des cartes », en raison de l'émergence d'une nouvelle offre de service - celle des coachs qui oblige à une redéfinition et à une nouvelle répartition des rôles professionnels. Ce contexte met en présence des acteurs sociaux qui tentent de préserver leurs acquis, de profiter de nouvelles opportunités ou de se distinguer. Ainsi présenté, ce contexte requiert la mobilisation d'une approche théorique qui peut rendre compte de cette dynamique. C'est dans cette perspective qu'ont été puisés des appuis aux travaux de chercheurs qui, d'une part, inscrivent les professions dans un monde social (Becker, 1988; Hughes, 1996) et, d'autre part, les considèrent comme des communautés de pratique (Wenger, 2005). Les domaines de la sociologie des professions et de l'apprentissage social proposent à cette fin des concepts sensibilisateurs (sensitive concepts ${ }^{2}$, Blumer, 1954) qui permettent d'appréhender les processus de constitution d'un groupe professionnel et de définition de sa pratique.

\section{La sociologie des professions}

Une perspective interactionniste des professions se centre sur les interactions qui animent un «monde social» (Becker, 1988, 2002; Hughes, 1996). Elle met en exergue les éléments de coordination de différents groupes professionnels dans leurs activités, indispensables pour rendre le service attendu (qui ne préjuge ni des accords ni des désaccords qui l'émaillent). Cette coordination se traduit par une organisation de la «coopération» pouvant donner lieu à des aménagements au sein du monde social, se traduisant par la mise en œuvre de «conventions » qui facilitent les interactions entre toutes les parties prenantes. Becker (1988) donne l'exemple de la musique, un domaine où il existe nombre de conventions qui balisent l'activité des différents intervenants; les fabricants réalisent des instruments de musique conventionnels pour lesquels les compositeurs écrivent leur musique dans un vocabulaire convenu, et les musiciens savent déchiffrer les partitions codées de manière habituelle; connues des différents partenaires, ces conventions font partie de la culture des groupes d'acteurs qui les intègrent dans leurs rôles, leur matériel, leur production. Par ailleurs, cette approche met en exergue la possible confrontation entre « règles de l'art» et «innovation ». Les premières sont le fruit du cheminement expérientiel réalisé par des générations d'acteurs qui exercent une pratique établie et dont chacun des partenaires reconnaît la pertinence et le déroulement; elles constituent des savoir-faire « durcis » d'une culture de métier, car s'étant révélés féconds en ce qu'elles permettent 
d'accomplir. Ainsi, lorsque les acteurs jouent leur rôle conformément aux conventions, ils sont considérés dans leur monde comme des professionnels intégrés qui réalisent une pratique selon des conventions communément admises (à l'instar des musiciens évoqués précédemment). La seconde renvoie à la nouveauté d'une pratique qui vient bousculer l'ordre établi par ses activités marginales, novatrices; elle peut être considérée comme un savoir-faire singulier de francs-tireurs. Donnant un exemple tiré du même domaine, Becker (1988) fait référence à un compositeur qui créerait une œuvre plus ou moins compatible avec les capacités d'exécution des interprètes et qui serait donc difficilement jouée, ou nécessitant des heures d'écoute et demandant alors au public une disponibilité inusitée. Ainsi, lorsque des acteurs bousculent des conventions, ils demandent aux autres de s'adapter et d'accepter leurs façons de faire. Un tel face-à-face peut conduire à l'abandon de l'innovation, à son intégration dans une pratique déjà existante ou à un réaménagement de l'espace social.

Cette première partie du cadre théorique fournit des concepts sensibilisateurs tels que «monde social », « coopération», «conventions », « règles de l'art» ou «innovation»; ceux-ci orientent l'interprétation des activités des coachs dans le cadre du mouvement d'expansion à l'externe qui amène la « redistribution des cartes » dans le monde de l'accompagnement au développement professionnel, tel qu'évoqué précédemment. Par leurs actions, les coachs visent à prendre une place dans le monde de l'accompagnement professionnel aux côtés de groupes professionnels déjà présents sur ce terrain. Leurs activités peuvent alors s'envisager au travers des réseaux d'influence mutuelle qui se constituent autour d'une pratique, des activités d'acquisition ou de défense des avantages, et des adaptations ou emprunts de conventions entre groupes professionnels.

\section{L'apprentissage social}

Une perspective interactionniste des communautés de pratique considère les interactions qui participent de la constitution d'un groupe professionnel. Elle met l'accent sur une "communauté » de membres engagés dans une même pratique qui apprennent au contact les uns des autres, définissant ainsi une pratique collective au travers du partage d'expériences singulières (Wenger, 2005). Cette coordination se traduit par la coconstruction d'un « répertoire partagé » de la pratique, soit des conventions qui la tissent - des savoir-faire donc - , des règles d'action ou des outils pertinents à sa réalisation. Ce bien commun est le fruit de la négociation de sens incessante qui a cours dans la communauté et qui contribue à en délimiter le territoire symbolique. 
Cette seconde partie du cadre théorique fournit des concepts sensibilisateurs tels que «communauté de pratique », "répertoire partagé », « conventions », « règles d'action », « territoire»; ceux-ci orientent l'interprétation des activités des coachs dans le cadre du mouvement d'organisation à l'interne qui vise à stabiliser les normes professionnelles, à rendre le service prévisible pour les clients, etc. Par leurs actions, les coachs ont pour objectif la constitution d'un groupe professionnel spécifique. Leurs activités peuvent alors s'envisager au travers des activités de coordination des membres, du partage des savoirs de la pratique qu'ils développent, de la création d'une identité de praticien-coach reconnue.

Le cadre théorique interactionniste ici bricolé - au sens ingénieux du terme -, à l'aune de travaux ancrés en sociologie des professions et en apprentissage social, conduit à aborder l'objet d'étude sous l'angle des processus qui animent le monde social dans lequel s'inscrit le coaching et ceux qui participent de la définition de la pratique des coachs. En cela, il s'agit bien d'un cadre théorique qui donne une "perspective » sur l'objet, et non d'un cadre conceptuel qui viendrait s'imposer aux données récoltées par la suite, prédéfinissant par avance les catégories d'analyse en «forçant» le terrain à se plier à un modèle théorique. Ce choix s'éloigne de la voie classique de la recherche en sciences de la gestion qui s'appuie sur des modèles théoriques de gestion qui servent de référents pour juger des pratiques de terrain. Le regard normatif généralement adopté vis-à-vis des activités des praticiens pose problème de notre point de vue, car les référents idéalisés ne permettent pas d'appréhender la complexité de la pratique des coachs, d'en saisir les nuances contextuelles.

Dès lors, la question de recherche capitalise sur toute la démarche déjà accomplie et formule une interrogation relative aux négociations qui ont cours dans le monde social pour la reconnaissance des coachs et de leur pratique et aux mouvements qui organisent l'activité professionnelle: Quels sont les processus de revendication d'un territoire de pratique qui animent un groupe professionnel en émergence, soit celui des coachs en gestion?

En cohérence avec les choix posés jusqu'ici, le cadre méthodologique présenté par la suite vise à créer un contexte susceptible de rendre compte des interactions qui mobilisent les acteurs dans la construction d'un sens autour de leur pratique.

\section{Le cadre méthodologique : une place centrale pour la question du sens}

La contextualisation de l'objet, sa « resocialisation» (Morrissette, 2010), suggère que des processus de négociation sont à l'œuvre dans la revendication 
d'un territoire de pratique, des processus qui, à la faveur des éclairages théoriques retenus, lient des groupes professionnels entre eux et en leur sein. Les choix méthodologiques doivent donc permettre d'appréhender la dynamique de négociation qui anime des groupes professionnels. En ce sens, il est nécessaire d'aménager un contexte qui suscite la négociation des significations.

\section{L'investigation de terrain : le point de vue négocié des coachs}

La recherche a impliqué la participation de cinq «francs-tireurs » (Becker, 1988), soit des praticiens qui innovent par rapport aux professionnels intégrés qui agissent selon les «règles de l'art». Plus spécifiquement, ce sont des praticiens à l'origine du mouvement de réaménagement de l'espace professionnel, des acteurs qui voulaient faire autrement et qui doivent faire preuve de créativité pour exister. Le recrutement s'est fait dans leur « réseau de sociabilité » existant (Gamson, 1992), sur une base volontaire, dans le respect de la diversité intrinsèque du groupe et de la représentativité des praticiens qui souhaitaient s'impliquer. Le groupe était donc constitué de cinq praticiens aux profils différents, que ce soit en termes d'âge, de sexe, de formation initiale, de carrière, d'ancienneté dans le groupe ou encore d'implication dans les activités du monde social (voir le Tableau 1).

Retenant du cadre théorique que la pratique fait l'objet d'une construction négociée entre les membres d'une communauté de pratique et en lien avec les membres d'autres groupes professionnels, ces praticiens ont été invités à partager des expériences autour de leurs activités professionnelles, dans le cadre de sept (7) entretiens collectifs. Ils ont eu à réfléchir entre pairs à propos d'un objet de préoccupation commune, passant alors d'un intérêt en commun à un intérêt commun au fil des rencontres (Anzieu \& Martin, 2000, citant Sartre $^{3}$ ). Ils ont ainsi débattu au sujet des activités liées à leur pratique en s'appuyant sur leurs préoccupations, en discutant de leurs représentations, en exprimant des croyances ou des conventions, en revendiquant des valeurs ou encore en interrogeant les tensions qu'ils expérimentent au cours de leurs activités professionnelles de coach.

Concrètement, les rencontres étaient marquées par le partage de récits de vie et de pratique (Bertaux, 2005; Desgagné, 2005) proposés par les praticiens, une méthode permettant l'explicitation d'une expérience vécue à partir de la narration des contextes, des actions en situation, des choix posés lors de l'intervention de coaching; ce partage entre pairs a donné à voir le sens qu'ils accordent à ces expériences, témoignant tant d'une histoire personnelle que collective (Demazière \& Samuel, 2010). Ainsi, selon une démarche déjà 
Tableau 1

Le profil des coachs participants

\begin{tabular}{|c|c|c|c|c|c|c|c|}
\hline Coach & Sexe & $\begin{array}{l}\text { Tranche } \\
\text { d'âge }\end{array}$ & $\begin{array}{l}\text { Discipline } \\
\text { d'études } \\
\text { (bac./ } \\
\text { maitrise) }\end{array}$ & $\begin{array}{l}\text { Activités } \\
\text { professionnelles } \\
\text { antérieures }\end{array}$ & $\begin{array}{l}\text { Ancienneté } \\
\text { en } \\
\text { coaching }\end{array}$ & Affiliation & Certification \\
\hline $\mathrm{C} 1$ & $\mathrm{~F}$ & $\begin{array}{l}50-60 \\
\text { ans }\end{array}$ & $\begin{array}{l}\text { Ensei- } \\
\text { gnement }\end{array}$ & $\begin{array}{l}\text { Ensei- } \\
\text { gnement } \\
\text { /Adminis- } \\
\text { tration scolaire }\end{array}$ & $\begin{array}{l}\text { Plus de } 10 \\
\text { ans }\end{array}$ & $\begin{array}{l}\mathrm{ICF}^{4} / \\
\mathrm{FICQ}^{5}\end{array}$ & $\begin{array}{l}\text { Master } \\
\text { Professional } \\
\text { Coach } \\
(M C C)\end{array}$ \\
\hline $\mathrm{C} 2$ & $\mathrm{~F}$ & $\begin{array}{l}30-40 \\
\text { ans }\end{array}$ & $\begin{array}{l}\text { Psycho- } \\
\text { éducation / } \\
\text { Relations } \\
\text { industrielles }\end{array}$ & $\begin{array}{l}\text { Commerce / } \\
\text { Conseil en } \\
\text { réadaptation }\end{array}$ & $\begin{array}{l}\text { Entre } 1 \\
\text { et } 5 \text { ans }\end{array}$ & $\begin{array}{l}\text { ICF / FICQ } \\
\text { (mandat } \\
\text { adminis- } \\
\text { tratif) }\end{array}$ & $\begin{array}{l}\text { Associate } \\
\text { Certified } \\
\text { Coach }(A C C)\end{array}$ \\
\hline $\mathrm{C} 3$ & $\mathrm{~F}$ & $\begin{array}{l}50-60 \\
\text { ans }\end{array}$ & $\begin{array}{l}\text { Mathéma- } \\
\text { tiques / } \\
\text { Sciences } \\
\text { économiques }\end{array}$ & $\begin{array}{l}\text { Adminis- } \\
\text { tration } \\
\text { publique }\end{array}$ & $\begin{array}{l}\text { Entre } 1 \\
\text { et } 5 \text { ans }\end{array}$ & ICF / FICQ & $\begin{array}{l}\text { Associate } \\
\text { Certified } \\
\text { Coach }(A C C)\end{array}$ \\
\hline $\mathrm{C} 4$ & $\mathrm{H}$ & $\begin{array}{l}30-40 \\
\text { ans }\end{array}$ & $\begin{array}{l}\text { Langues / } \\
\text { Anthro- } \\
\text { pologie }\end{array}$ & Formation & $\begin{array}{l}\text { Entre } 5 \\
\text { et } 10 \text { ans }\end{array}$ & $\begin{array}{l}\text { SICPNL }^{6} \\
\text { (mandat } \\
\text { adminis- } \\
\text { tratif) }\end{array}$ & $\begin{array}{l}\text { Post Maitre } \\
\text { PNL }\end{array}$ \\
\hline C5 & $\mathrm{H}$ & $\begin{array}{l}30-40 \\
\text { ans }\end{array}$ & $\begin{array}{l}\text { Adminis- } \\
\text { tration des } \\
\text { affaires }\end{array}$ & Marketing & $\begin{array}{l}\text { Entre } 1 \\
\text { et } 5 \text { ans }\end{array}$ & ICF / FICQ & $\begin{array}{l}\text { Associate } \\
\text { Certified } \\
\text { Coach }(A C C)\end{array}$ \\
\hline
\end{tabular}

éprouvée dans le cadre de travaux précédents (voir notamment Morrissette, 2011), un premier temps était consacré à des récits d'expériences individuels, à partir du «faire individuel »; un second temps était dédié aux débats collectifs suscités par les récits préalablement livrés afin de favoriser l'explicitation des activités et la négociation de leurs significations entre pairs, vers un « faire collectif $»$.

Les récits de pratique ont été choisis en fonction de trois thématiques, inspirées du cadre théorique par le truchement de concepts sensibilisateurs et d'une recherche antérieure (Guignon, 2006). En outre, elles ont été avalisées par une activité exploratoire de type remue-méninge menée avec les praticienscoachs participants. L'une de ces thématiques, Apprendre, avait pour but de solliciter récits et débat autour des éléments qu'ils envisageaient comme 
représentatifs de leurs apprentissages; elle a mené à l'approfondissement des éléments ayant trait aux parcours individuels des coachs, mais aussi aux dimensions plus collectives de l'apprentissage d'une pratique de coaching. Une autre thématique, Intervenir, mettait l'accent sur des aspects considérés comme significatifs, par exemple des pratiques exemplaires ou des pratiques à éviter ou encore des façons de faire valorisées; elle a conduit à approfondir des éléments de la culture du groupe, des moyens de rendre la pratique spécifique pour se différencier des autres groupes. Une dernière thématique, Développer, ciblait des éléments vus comme caractéristiques de leurs environnements de travail pour le déploiement de leurs activités; elle a mis en lumière le réseau social au sein duquel les coachs disent évoluer, de même que la nature de la coopération prenant place entre les divers groupes en présence.

Les rencontres ont favorisé une coconstruction de sens qui pouvait s'inscrire selon trois «zones » du territoire revendiqué, telles que définies par Morrissette (2010): une zone partagée, se traduisant dans la présence de consensus renforçant la culture du groupe, une zone admise, tissée de positions plurielles reconnues comme pertinentes dans un monde professionnel en définition, et une zone contestée, donnant à voir des désaccords persistants concernant des façons de faire ou de voir la pratique, soit autant de réflexions réalisées en vue d'expliquer ou de convaincre un public de pairs (Clot \& Faïta, 2000). Ainsi, d'une part, aborder le terrain selon une perspective interactionniste conduit à déployer une démarche pour permettre aux acteurs « de mettre en mots leur expérience » (Guignon \& Morrissette, 2006); il est dès lors question de s'intéresser au sens que les acteurs accordent à leurs activités, à la façon dont ils «définissent la situation » (Thomas, 1923). D’autre part, cela conduit à favoriser le métissage des points de vue pour leur permettre de négocier les significations qu'ils y attachent. En outre, les entretiens collectifs constituent des occasions privilégiées de prendre connaissance des questionnements issus du milieu, plutôt que de lui imposer une vision cadrée en amont ou en aval par un dispositif de recherche qui aurait ses propres visées normatives.

\section{L'approche analytique : un projet théorique qui insuffle du sens à la parole des coachs}

Le dispositif de recherche a débouché sur un corpus qui résulte d'interactions, de négociations de sens autour de la pratique du coaching par des praticiens dont c'est l'activité professionnelle principale. En effet, les expériences relatées durant les récits individuels étaient élaborées par les coachs dans le contexte social de l'entretien collectif au cours duquel ils se dévoilent, s'accueillent et construisent en commun, les pairs servant de cadre de référence les uns pour les 
autres. Quant aux débats collectifs, ils permettaient aux coachs de faire appel aux expériences narrées précédemment (dans la même rencontre ou lors de rencontres passées) pour asseoir leurs commentaires ou croiser différents points de vue. Ainsi, l'ensemble des verbatim transcrits met en évidence le produit collectif de la réflexion. Sous cet angle, l'approche analytique vise l'interprétation de la parole des coachs sollicités, pour appréhender la revendication qu'ils font d'un territoire de pratique, selon une posture dite analytique $^{7}$ (Demazière \& Dubar, 2004).

La perspective interactionniste privilégiée depuis le début de la recherche se traduit dans cette phase par le choix d'une approche inductive pour donner sens aux entretiens collectifs qui sont des dialogues entre pairs à propos de sujets centrés sur leurs expériences. Dans cette perspective, le chercheur interprète les données colligées pour aller plus loin que le sens donné par les acteurs à leur expérience, pour construire une compréhension autour d'un autre "phénomène » que celui décrit par les praticiens, en fonction du projet théorique qui est matérialisé par la question de recherche, clé du sens et de l'interprétation du corpus recueilli.

Il existe un certain nombre de principes et d'étapes génériques nécessaires à une analyse inductive, comme le relève Thomas (2006), et ceuxci ont été mises en œuvre pour réaliser la présente recherche. La première vise à préparer les données, par exemple des verbatim. La seconde consiste à procéder à une lecture approfondie et répétée des textes disponibles pour recenser des thèmes et catégories possibles. La troisième étape conduit à regrouper sous des thèmes porteurs de sens des parties de verbatim qui semblent pouvoir éclairer l'objet de la recherche ${ }^{8}$. L'identification des catégories d'analyse fait l'objet de la quatrième étape et est le résultat d'un travail d'affinage des différents thèmes pour aboutir à des catégories et à des sous-catégories qui, au sein d'un sens générique partagé, amènent des distinctions qui enrichissent le modèle en cours de construction. Au final, il s'agit de proposer une nouvelle compréhension ou une nouvelle théorie ancrée dans l'empirie qui éclaire l'objet à l'étude, qui apporte une réponse à la question de la recherche négociée au-delà du sens premier porté par les propos des participants. C'est ainsi qu'ont été dégagés des processus de la revendication du territoire de pratique, comme nous l'illustrons en explicitant un exemple.

Des résultats de recherche : un nouveau point de vue sur la définition d'un groupe professionnel et de sa pratique

L'investigation de terrain ainsi que l'approche analytique ont produit un matériau qui relate la négociation du sens des activités menées par les coachs 
dans leur monde social, un ajustement de la définition de la pratique vu au travers des échanges entre pairs. Ce matériau est exploité en interprétant ce sens que les praticiens donnent à leurs activités avec pour objectif de rendre compte de la constitution du groupe professionnel des coachs, au travers de processus qui visent à appréhender le mouvement vers ce projet d'établissement professionnel. La question leitmotiv durant les lectures de verbatim était « qu'est-ce qu'ils font ensemble pour se constituer en tant que groupe? ». Cela a conduit à dégager quatre processus de revendication animés par les coachs : « la projection dans un nouveau paradigme », « la construction identitaire collective », «l'établissement d'un réseau de coopération » et «la normalisation d'une pratique ».

En synthèse, le premier processus, «la projection dans un nouveau paradigme ", permet d'éclairer la coconstruction d'une représentation de l'évolution des organisations en vue d'accueillir l'innovation que propose le coaching en gestion. Il s'est agi pour les coachs d'opposer une «ancienne mentalité » et un «nouveau paradigme » au travers d'une vision des organisations, des relations de travail, des modes d'accompagnement. Ce faisant, les coachs en sont venus à décrire un monde social reconfiguré pour exister.

Le deuxième processus, « la construction identitaire collective », rend compte du partage et de la consolidation de marqueurs identitaires que les coachs vivent dans le cours de leurs activités. Ils font ainsi référence à des activités qui leur permettent de se reconnaître des maitres, de créer des artéfacts et des formes de rituels rassembleurs, des éléments de transfert de savoir qui dénotent l'affiliation identitaire. C'est dans ces interactions que les coachs construisent une identité commune pour se [faire] (re)connaître.

Le troisième processus, "l'établissement d'un réseau de coopération », renvoie à l'établissement d'alliances stratégiques, aux choix des supporteurs (clients dans la haute direction et gestionnaires), aux partenariats acceptables (consultants) ou non (naturopathes), à la prise en compte des concurrents (psychologues), à la reconnaissance des affiliés selon certaines modalités d'appartenance (coachs internes, gestionnaires, service des ressources humaines ${ }^{9}$ ). Bref, les coachs tissent un réseau pour s'implanter, comme cela sera illustré ci-dessous.

Enfin, le quatrième processus, «la normalisation d'une pratique », éclaire les accords auxquels les acteurs parviennent concernant l'usage de modèles existants, les balises qu'ils tirent de l'innovation pour cadrer leur agir ainsi que les aménagements des principes d'action pour s'adapter aux contextes. Il s'agit pour eux de mettre à disposition de l'innovation les 
connaissances et expériences acquises antérieurement, d'emprunter des modèles et des outils ayant fait leur preuve pour commencer à construire un répertoire de pratique qui pourra être partagé, mais de donner un tour de main spécifique à ces façons de faire empruntées respectueuses des valeurs qui soustendent l'innovation. Ils fabriquent dès lors une pratique spécifique.

À titre d'illustration, le troisième processus, «l'établissement d'un réseau de coopération», aborde les négociations rendues nécessaires et réalisées avec différents partenaires identifiés pour prendre une place dans le monde social. Les coachs ont ainsi dressé la cartographie d'un réseau pour s'implanter, des alliances dont se méfier ou des coalitions jugées pertinentes dans la perspective de s'établir durablement.

S'établir, c'est avant tout développer des liens avec les groupes professionnels déjà existants. L'espace socioprofessionnel que les coachs investissent est occupé par de nombreux intervenants. Les coachs y négocient donc leur place en établissant la légitimité de leur pratique professionnelle, en définissant ce qui les distingue des autres. En position de francs-tireurs, ils ont besoin de se trouver des alliés. Ils vont rechercher par exemple la coopération de clients et de prescripteurs qui trouvent un intérêt à la pratique qu'ils proposent.

C5 Je parlais avec quelqu'un des ressources humaines dans la fonction publique cette semaine, la directrice vient de déléguer à un de ses professionnels de se faire une tête sur le coaching

C5 quelle place ils vont donner au coaching

C3 /ouais

C5 //versus le mentorat et la formation; la formation ça va de soi, le mentorat, ils ont acheté l'idée, ça ne marche pas encore, mais ils ont acheté une idée; le coaching, ils savent pas quoi faire avec ça, [...] ça fait que moi innocemment, mais en même temps pour lui vendre l'idée, je lui ai dit « j'imagine que quand vous allez vous orienter vers le coaching, le fait de faire affaire avec des coachs professionnels certifiés par la Fédération internationale, ça va sûrement être un nouveau critère [...] aussi, n'est-ce pas », point d'interrogation; elle ne le savait pas vraiment, mais elle m'a dit, « si vous avez de l'information là-dessus »

$\mathrm{C} 2$ //oui

C5 // « quels sont les bénéfices »

C3 //c'est ça 
C5 les bénéfices, je venais de finir mon argumentaire, je le lui ai fait parvenir dans l'heure qui a suivi, là, et quelque part, je pense qu'on a une possibilité d'influence.

(R2_265-283)

Ils vont établir des partenariats avec des professionnels reconnus, bien qu'a priori ceux-ci puissent apparaître comme des concurrents. Par exemple, ils vont voir dans un rapprochement avec des consultants une sorte de complémentarité.

C3 il y a des firmes de ressources humaines, partenaires, des firmes de conseil, [...] une firme comme ça, peut décider d'avoir une offre de coaching, et signer des ententes de partenariat avec des sous-traitants qui sont coachs

C4 ah oui

C5 en fait, c'est un partenaire

//quand tu

C2 //oui

C5 fais partie des partenaires

//et c'est un concurrent quand tu es exclu des partenaires.

(R6_2775-2796)

Par ailleurs, les coachs doivent se préoccuper de la légitimité des professionnels qui se forment, prennent la carte d'une fédération ou affichent des compétences diverses. Les coachs ne voient pas dans chacun de ces professionnels des pairs. Ainsi, ils vont se définir eux-mêmes, coachs externes, indépendants des organisations pour lesquelles ils peuvent réaliser des mandats de coaching; ils vont attribuer une qualification de coach à certains intervenants, les coachs internes, tout en limitant la définition de leur mandat; ils vont la refuser catégoriquement à d'autres, les gestionnaires ou les conseillers aux ressources humaines, mais promouvoir le coaching comme outil de gestion, encourageant la diffusion et l'intérêt pour le coaching.

C5 okay, mon point ce n'était pas de dire que je ne trouvais pas ça correct, mais c'était de délimiter jusqu' où va le coaching interne dans les organisations; donc, même une entreprise qui croit au coaching interne, qui a investi pour faire former quelqu'un, dans ce cas-là c'est un bel exemple, il reste quand même un marché de hauts dirigeants qui va à l'externe

C3 oui, puis je trouve que le découpage n'est pas si mal, si j'avais à faire un découpage rapidement, moi c'est à peu près comme ça que je le 
ferais, puis il ne serait peut-être pas égal, dépendamment des postes, il y a un poste stratégique là, je dis non, lui il ne sera pas en interne, peu importe

C2 c'est intéressant ce que tu dis, parce que là tu parles dépendamment des postes, il y a des besoins aussi, c'est autre chose

C3 les besoins c'est autre chose aussi.

(R2_982-993)

Ceci étant dit, la négociation de l'espace avec les autres intervenants permet aussi d'envisager la pratique du coaching elle-même. Il est possible de dégager au moins deux espaces de pratique (Guignon, 2011). L'un, que les coachs s'octroient sans partage - la zone réservée de leur innovation. Ils y associent une légitimité qui établit leur spécificité. En se réservant certaines compétences, ils définissent la pratique, ce qu'elle recouvre, sa mise en œuvre, le service rendu [original] auquel des clients potentiels peuvent recourir. L'autre, que les coachs concèdent sous condition - la zone partagée de leur innovation. Ils y associent une stratégie d'établissement dans le contexte de leur émergence. En envisageant que certaines activités, dans certains contextes, peuvent être exercées par d'autres praticiens qu'eux-mêmes, ils donnent à connaître des éléments de la pratique qui demandent des ajustements, des modèles d'intervention qui peuvent s'écarter de ce qu'ils estiment être une pratique "pure », c'est-à-dire de l'ordre de l'idéal ${ }^{10}$. Ainsi, les coachs nous apprennent qu'il existe des cibles et des formes de coaching différentes, mais pas pour autant un développement de pratiques aléatoires. En négociant leur réseau de coopération dans le monde social, ils précisent leur pratique, sa portée, ainsi que les praticiens qu'ils considèrent comme légitimes dans leurs activités.

Ainsi, les frontières entre les groupes professionnels qui réalisent des activités d'accompagnement au développement professionnel, et surtout leurs pratiques, sont moins étanches que pourraient le laisser croire certains modèles théoriques appliqués dans une perspective top-down (Joo, 2005; Natale \& Diamante, 2005). L'analyse du processus d'établissement d'un réseau de coopération par les coachs suggère plutôt que ces frontières sont plus ou moins ouvertes, selon les intérêts négociés des divers acteurs présents dans le monde social. La recherche a dès lors intérêt à laisser remonter du terrain (bottom-up) la compréhension dont sont porteurs les acteurs qui agissent en contexte, afin de prendre appui sur les négociations dont ils font état et qui modèlent la pratique pour comprendre celle-ci en tenant compte des enjeux qui lient les groupes en présence. 
En somme, le cadre méthodologique, tant en regard de l'investigation de terrain que de l'approche analytique utilisée, tire profit de l'approche qualitative déployée à travers la mobilisation d'une perspective interactionniste. Il a permis d'appréhender la reconfiguration continue qui anime la mosaïque professionnelle, tels que dégagée du cadre contextuel. Il a aussi conduit au recueil, auprès de praticiens, des récits de pratique et des éléments de négociation à même de renseigner les processus qui façonnent un groupe qui veut établir sa pratique dans un monde social. De même, il a favorisé l'interprétation d'activités réalisées par des coachs pour faire (re)connaître leur pratique comme spécifique, à côté de celles déjà bien établies dans le domaine de l'accompagnement au développement professionnel. Ces différents choix mettent en relief une distance par rapport à la voie classique de la recherche en sciences de la gestion qui s'appuie plus traditionnellement sur des méthodologies dont l'épistémologie, de nature positiviste, renvoie à l'usage de variables prédéterminées et vise à valider ou invalider des hypothèses préétablies. En l'espèce, une façon traditionnelle d'investir le terrain aurait pu consister à faire passer un questionnaire, plus ou moins conséquent, à une population plus ou moins importante de coachs. Mais, ce faisant, le sens est impulsé par le chercheur dans la formulation qu'il fait des questions. Dans le cadre de la recherche ici présentée (Guignon, 2012), c'est la parole des acteurs qui a été privilégiée, celle des praticiens confrontés au quotidien à ce contexte d'émergence et dont eux seuls peuvent rendre compte du sens qu'ils y accordent.

\section{Discussion conclusive}

La recherche adopte traditionnellement une approche de type research down dans nombre de domaines, dont les sciences de la gestion (Guignon \& Morrissette, 2006). Souvent menée selon une approche quantitative, elle mobilise des modèles théoriques qui précontraignent variables et questions, ou comptabilisent des éléments décontextualisés; elle vise à en vérifier la mise en œuvre adéquate ou le bon rendement. Dans une telle démarche, les acteurs et leurs activités sont souvent pointés comme déficitaires en regard des attentes fixées préalablement à l'appréhension du terrain. Sans remettre en question l'apport scientifique de telles recherches, il n'en demeure pas moins que certains phénomènes ne peuvent être captés et interprétés par ces démarches. L'indéterminé et la complexité des situations professionnelles, les processus de (re)composition continue à travers les interactions qui tissent les mondes sociaux, les interdépendances et les jeux d'influence mutuelle, les enjeux sousjacents au sens véhiculés par des activités, sont autant d'éléments qui prennent 
part à une dynamique que des approches qualitatives appréhendent de façon plus féconde.

En conséquence, délaisser la voie classique de la recherche en sciences de la gestion pour embrasser une perspective interactionniste a permis d'appréhender un phénomène en émergence, qui n'est ni homogène ni stabilisé, et ce, sans que cela soit un problème, mais plutôt une source de réflexion. De fait, en portant le regard sur les interactions entre acteurs, il a été possible de mettre en lumière de cette façon le social «en train de se faire », soit des processus mobilisés par des acteurs pour un projet collectif, ici l'établissement d'une profession. Ces processus, documentés sur la base des expériences négociées entre pairs, donnent à connaître, par exemple, le contexte des activités auxquelles ils participent tel qu'ils le conçoivent et, par là même, nous renseignent à propos des liens qu'ils entretiennent avec leurs partenaires.

Ainsi, au vu de la recherche, la façon dont les clients et prescripteurs qui peuvent faire appel au coaching, plutôt qu'à d'autres types d'accompagnement au développement professionnel, donne aux coachs de nouvelles balises pour penser leur positionnement dans les entreprises; certaines d'entre elles élaborent des grilles croisant des profils de coachs externes et internes certification, niveau, expériences - avec des objectifs de développement ou de niveaux hiérarchiques pouvant être pris en charge. Leur réflexion à propos de la pratique, aussi adressée dans la recherche en termes de modèles d'intervention et d'outils, offre de nouvelles perspectives pour envisager sa prestation, ses prérequis et ses cibles (la définition d'une coresponsabilité tripartite par exemple est centrale dans le concept de l'intervention en coaching). Ce type de résultats de recherche est donc significatif pour le domaine de la gestion des entreprises et des ressources humaines, permettant d'identifier plus finement comment employer des coachs, pour quoi faire.

En outre, en s'attardant aux activités des coachs, mais surtout à ce qu'ils en disent et à ce qu'ils en négocient, entre pairs et avec leurs partenaires, il a été possible de mettre également en lumière comment la négociation d'un territoire symbolique par un groupe professionnel affecte la définition de sa pratique. De fait, les coachs, à titre d'acteurs sociaux compétents (Giddens, 1987), sont capables de mobiliser des ressources et de mettre en œuvre des stratégies pour réaliser leur projet d'établissement professionnel. La recherche a permis de rendre compte que, pour ce faire, ils en viennent à accepter des accords pragmatiques et à choisir des alliances. En agissant de la sorte, ils négocient différents compromis qui profanent en quelque sorte cet espace professionnel symbolique, cette zone réservée de leur innovation, en dénaturant l'image spécifique de la profession. C'est ainsi qu'ils vont par exemple 
promouvoir le coaching pour les coachs internes (une pratique privée de certains attributs) ou comme outil pour les gestionnaires (une pratique reconfigurée pour une autre visée). À nouveau, ce type de résultats de recherche est significatif pour le domaine, permettant de discerner différents types dans la pratique, délibérément acceptés, et non une pratique disparate restant à uniformiser, à codifier.

À la lumière de cette contribution, il est possible d'avancer que l'usage de démarches de recherches qualitatives en sciences de la gestion est une voie peut-être encore «originale», comme le définit l'introduction de ce numéro spécial, mais pleine de promesses. La recherche qualitative et les points de vue qu'elle met en exergue doivent être envisagés comme complémentaires, étendant la pertinence sociale des recherches menées à propos d'un objet d'étude. Finalement, la multiplicité des regards vient enrichir la compréhension que l'on construit d'un objet d'étude, tout comme parcourir différents sentiers de randonnée permet de multiplier les expériences que l'on a d'un même lieu.

\section{Notes}

${ }^{1}$ L'auteure remercie le Conseil de recherche en sciences humaines du Canada (CRSH) ainsi que le Fonds québécois pour la recherche en sciences sociales (FQRSC) dont les financements, sous la forme de bourses d'études ont rendu possible la réalisation de la thèse à partir de laquelle est tiré cet article.

${ }^{2}$ Tel que l'explicite Piette (1996), Blumer envisage deux types de concepts : des concepts « définitifs » qui s'appuient sur des représentations symboliques d'objets (des classes d'objets) dont les attributs sont déterminés de manière prescriptive; des concepts «sensibilisateurs» qui fournissent un sens général, une référence, une orientation. En ce sens, des concepts sensibilisateurs orientent la compréhension, tandis que des concepts définitifs commandent une analyse.

${ }^{3}$ Sartre, J. P. (1960). Critique de la raison dialectique. Paris : Gallimard.

${ }^{4}$ ICF : International Coach Federation.

${ }^{5}$ FICQ : Fédération internationale des coachs du Québec.

${ }^{6}$ SICPNL : Société internationale des coachs en PNL.

${ }^{7}$ C'est la façon dont ces auteurs nomment l'une des trois postures d'analyse qu'ils identifient : restitutive, démonstrative et analytique.

${ }^{8}$ Le classement émergeant à cette étape était à nouveau supporté par les concepts sensibilisateurs acquis dans le cadre théorique, reprenant des aspects définissant les groupes professionnels, les mondes sociaux, les communautés de pratique. À titre d'exemple, des thèmes [et sous-thèmes] tels la légitimité [le sale boulot, les concurrents], l'identité [le nom, les attributs du nom], etc. ont pu émerger et servir de 
base au travail d'analyse, autant d'éléments qui orientaient le regard posé et le sens construit pour répondre au projet analytique.

${ }^{9} \mathrm{D}$ 'après les coachs, les naturopathes, qui se montrent intéressés par un rapprochement avec les coachs relativement aux avantages qu'ils en tireraient, ne sont pas des partenaires acceptables, notamment parce qu'ils s'inscrivent dans le domaine de la santé, une image à laquelle les coachs souhaitent ne pas être associés.

${ }^{10}$ Par exemple, une valeur primordiale dans l'établissement d'une relation de coaching passe par la confidentialité. Or, du point de vue des coachs externes, le coaching interne pourrait entrainer la perception d'un risque dans le fait de se confier à un intervenant lié à l'entreprise. C'est donc la garantie du secret qui est en jeu dans ce modèle d'intervention qui s'écarte de l'idéal.

\section{Références}

Abbott, A. (1999). Department and discipline: Chicago sociology at one hundred. Chicago : University of Chicago Press.

Abbott, G. N., Stening, B. W., Atkins, P., \& Grant, A. M. (2006). Coaching expatriate managers for success: adding value beyond training and mentoring. Asia Pacific Journal of Human Resources, 44(3), 295-317.

Anzieu, D., \& Martin, J.- Y. (2000). La dynamique des groupes restreints. Paris : Presses universitaires de France.

Arfel, F. (2002). Qui sont les coaches? Actualité de la formation permanente, $178,32-34$.

Audet, J., \& Couteret, P. (2005). Le coaching entrepreneurial : spécificités et facteurs de succès. Journal of Small Business \& Entrepreneurship, 18(4), 471-490.

Becker, H. S. (1988). Les mondes de l'art. Paris : Flammarion.

Becker, H. S. (2002). Les ficelles du métier. Comment conduire sa recherche en sciences sociales. Paris : La découverte.

Berglas, S. (2002). The very real dangers of executive coaching. Harvard Business Review, 80(6), 86-93.

Bertaux, D. (2005). L'enquête et ses méthodes. Le récit de vie. Paris : Armand Colin.

Bloch, S. (1996). Coaching tomorrow's top managers. The Journal of Workplace Learning, 8(5), 30-32. 
Blumer, H. (1954). What is wrong with social theory? American Sociological Review, 19(1), 3-10.

Boltanski, L., \& Chiapello, E. (1999). Le nouvel esprit du capitalisme. Paris : Gallimard.

Brunel, V. (2004). Les managers de l'âme: le développement personnel en entreprise, nouvelle pratique de pouvoir. Paris : Découverte.

Chapoulie, J.- M. (2001). La tradition sociologique de Chicago, 1892-1961. Paris : Éditions du Seuil.

Clot, Y., \& Faïta, D. (2000). Genre et style en analyse du travail. Concepts et méthodes. Travailler, 4, 7-42.

Crozier, M. (1994). L'entreprise à l'écoute : apprendre le management postindustriel. Paris : Éditions du Seuil.

Desgagné, S. (2005). Récits exemplaires de pratique enseignante. Analyse typologique. Québec : Presses de l'Université du Québec.

Demazière, D., \& Dubar, C. (2004). Analyser les entretiens biographiques. L'exemple des récits d'insertion. Québec : Presses de l'Université Laval.

Demazière, D., \& Samuel, O. (2010). Inscrire les parcours individuels dans leurs contextes. Temporalités, $11 . \quad$ Repéré à http://temporalites.revues.org/1167

Desrosières, A. (2008). Gouverner par les nombres. L'argument statistique. Paris : Presses de l'École des mines.

Eaton, J., \& Brown, D. (2002). Coaching for a change with Vodafone. Career development international, 7(5), 284-287.

Feldman, D. C., \& Lankau, M. J. (2005). Executive coaching: a review and agenda for future research. Journal of management, 31(6), 829-848.

Frisch, M. H. (2005). Extending the reach of executive coaching : the internal coach. Human Resource Planning, 28(1), 23.

Gamson, W. A. (1992). Talking politics. New York : Cambridge University Press.

Garman, A. N., Whiston, D. L., \& Zlatopec, K. W. (2000). Media perception of executive coaching and the formal preparation of coaches. Consulting Psychology Journal : Practice \& Research, 52(3), 201-205.

Giddens, A. (1987). La constitution de la société. Paris : Presses universitaires de France. 
Gray, D. E. (2006). Executive coaching: towards a dynamic affiance of psychotherapy and transformative learning processes. Management Learning, 37(4), 475-497.

Guignon, S. (2006). Des savoirs pratiques construits à partir de récits d'intervention de coachs en entreprise (Mémoire de maitrise inédit). Université Laval, Québec, QC.

Guignon, S. (2011). Le territoire de pratique revendiqué par des coachs en gestion. Une analyse interactionniste. Recherche qualitative, 30(1), 200223.

Guignon, S. (2012). La revendication de territoire des coachs en gestion. Une approche interactionniste pour rendre compte de l'émergence d'un groupe professionnel (Thèse de doctorat inédite). Université Laval, Québec, QC.

Guignon, S., \& Morrissette, J. (2006). Quand les acteurs mettent en mots leur expérience. Recherches qualitatives, 26(2), 19-36.

Hall, D. T., Otazo, K. L, \& Hollenbeck, G. P. (1999). Behind closed doors : what really happens in executive coaching. Organizational dynamics, 27(3), 39-53.

Hughes, E. C. (1996). Le regard sociologique. Essais choisis. Paris : Éditions de l'École des Hautes Études en Sciences Sociales.

Joo, B. K. (2005). Executive coaching: a conceptual framework from an integrative review of practice and research. Human Resource Development Review, 4(4), 462-488.

Kilburg, R. (2000). Executive coaching : developing managerial Wisdom in a world of chaos. Washington, DC : American Psychological Association.

Latour, B. (1999). Politiques de la nature. Comment faire entrer les sciences en démocratie. Paris : La Découverte.

Malarewicz, J. A. (2002). Systémique et entreprise: coaching, relations interpersonnelles, changement. Paris : Village Mondial/Pearson Education France.

Malarewicz, J. A. (2003). Réussir un coaching: grâce à l'approche systémique. Paris : Village Mondial/Pearson Éducation France.

Morrissette, J. (2009). Une resocialisation de l'évaluation. Revue canadienne des jeunes chercheurs en éducation / Canadian Journal for New Scholars in Education (CJNSE/RCJCE), 1(2). Repéré à http://www.cjnsercjce.ca/ojs2/index.php/cjnse/article/view/57/63. 
Morrissette, J. (2010). Une perspective interactionniste : un autre point de vue sur l'évaluation des apprentissages. SociologieS. Repéré à http://sociologies.revues.org/3028

Morrissette, J. (2011). Ouvrir la boîte noire de l'entretien de groupe. Recherches qualitatives, 29(3), 7-32.

Morrissette, J., Guignon, S., \& Demazière, D. (2011). De l'usage des perspectives interactionnistes en recherche. Recherches qualitatives, 30(1), $1-9$.

Morrissette, J., \& Legendre, M.- F. (sous presse). Les enjeux éthiques et politiques de l'évaluation en éducation. Québec : Presses de 1'Université Laval.

Natale, S. M., \& Diamante, T. (2005). The five stages of executive coaching : better process makes better practice. Journal of Business Ethics, 59, 361374.

Paul, M. (2004). Ce qu'accompagner veut dire. Carriérologie, 9(1-2), 121-144.

Parissier, C., \& Audet, M. (Éd.). (2013). La recherche qualitative dans les sciences de la gestion. De la tradition à l'originalité. Recherches qualitatives, 32(2).

Piette, A. (1996). Ethnographie de l'action. L'observation des détails. Paris : Métailié.

Rappin, B. (2006). Le coaching ou la transparence intégrale. Revue internationale de psychologie, 12(27), 203-220.

Sartre, J.- P. (1960). Critique de la raison dialectique. Paris : Gallimard.

Sperry, L. (2008). Executive coaching: an intervention, role function, or profession? Consulting Psychology Journal : Practice \& Research, 60(1), 33-37.

Thomas, D. R. (2006). A general inductive approach for analyzing qualitative evaluation data. American Journal of Evaluation, 27(2), 237-246.

Thomas, W. I. (1923). The unadjusted girl with cases and standpoint for behavior analysis. Boston : Little Brown and Company.

Vernazobres, Ph. (2006). Le coaching, révélateur et vecteur de nouveaux modes de régulation sociale en entreprise. Revue internationale de psychologie, 12(27), 179-201.

Wasylyshyn, K. M. (2003). Executive coaching : an outcome study. Consulting Psychology Journal : Practice \& Research, 55(2), 94-106. 
102 ReChERChEs Qualit ATIVES / Vol. 32(2)

Wenger, E. (2005). La théorie des communautés de pratiques. Apprentissage, sens et identité. Québec : Presses de l'Université Laval.

Sylvie Guignon (Ph.D.) est consultante auprès de l'Université Laval dans le cadre du développement d'une communauté de pratique des directeurs de recherche; elle détient un doctorat et une maitrise en psychopédagogie délivrés par cette institution. Elle a auparavant occupé différents postes au sein d'entreprises et d'organismes gouvernementaux. Ses travaux puisent à l'interactionnisme, aux communautés de pratique et à la sociologie des professions.

Joëlle Morrissette (Ph.D.) est professeure adjointe à la Faculté des sciences de l'éducation de l'Université de Montréal. Elle s'investit particulièrement en recherche méthodologique, selon une perspective interactionniste qui conduit à saisir, de manière compréhensive, les significations des pratiques professionnelles pour ceux et celles qui les mettent en ouvre, et à repérer les processus d'ajustement et de négociation au principe de leur régulation. 